\title{
Efficient and Reliable Method of Time Distributed Media Access Control for loT Devices
}

\author{
VALENTYN FAYCHUK ${ }^{1}$, DARIUSZ MROZEK ${ }^{2}$, BOHDAN STRYKHALIUK ${ }^{3}$, \\ OREST LAVRIV ${ }^{4}$, OLGA SHPUR ${ }^{1}$ \\ ${ }^{1}$ Department of Telecommunication, Lviv Polytechnic National University, Ukraine, (e-mail: faitchouk.valentyn@gmail.com, olha.m.shpur@lpnu.ua) \\ ${ }^{2}$ Department of Applied Informatics Silesian University of Technology, Gliwice, Poland, (e-mail: dariusz.mrozek@polsl.pl) \\ ${ }^{3}$ Department of Radioelectronic Devices and Systems, Lviv Polytechnic National University, Ukraine, (e-mail: bohdan.m.strykhaliuk@lpnu.ua) \\ ${ }^{4}$ Department of Electronics and Information Technology, Lviv Polytechnic National University, Ukraine, (e-mail: orest.a.lavriv@lpnu.ua)
}

Corresponding author: Olga Shpur (e-mail: olha.m.shpur@lpnu.ua).

\begin{abstract}
: ABSTRACT Wireless multi-hop communication is used widely today though there are many uneven issues related to the process of organizing the transmission, e.g., collisions, caused by simultaneous transmission of two objects that are equally distant toward opposite directions from the receiver, and so on. To address such cases, there are solutions, such as CSMA or NB-IoT. The point is that common solutions propose to use an arbiter (e.g. AP), which controls transmission between hops. The main benefit of the approach is the simplicity, but it lacks flexibility. In other cases, if the arbiter is absent, common methods use intense sequential access to the spectrum and queued control over the radio resource. However, these techniques have selective spectrum usage, i.e., the reserved bandwidth is used only partially. In separate cases, these may impact security and stability as well. The paper discusses a new concept: get rid of arbiter and organize an Ad-hoc network, where resources are dynamically distributed, depending on the needs. The only limitation is that the medium must be able to hold enough bandwidth to organize a set of separate carriers, we call tunnels, for parallel communication. Normally, tunnels are uniformly occupied by hops. So, the primary goal of this work is to develop a new principle for tunnel selection by two or more nodes and define the impact of its parameters onto the resulting performance. The tunnel is then used as a detached medium, shared exclusively between these nodes for communication. Thus, two regimes can be outlined: the regime of picking an appropriate tunnel and regime of communication. In the paper, we will discuss the regime of picking the tunnel, i.e., present the principle that allows doing that, study the influence of its technical parameters on its tactical parameters, and define best conditions for its operation. As a result, we proposed method of time distributed media access control for picking carrier (tunnel), which is more flexible compared to the current solutions as it does not require an arbiter and allows using tunnels simultaneously as well as change them dynamically.
\end{abstract}

: KEYWORDS CSMA/CA, CSMA/CD, ad-hoc network, SNR, heterogeneous IoT networks.

\section{INTRODUCTION}

$\mathrm{T}$ HERE are two common approaches to solving the problem of providing communication in a resourceslimited medium: to use the available bandwidth asynchronously, which defines continuous competition between nodes for the shared medium; and to divide bandwidth into small parts that are used as separate peer-to- peer links. The asynchronous approach generates transmission peaks because it assumes transmitting all the data within its bandwidth availability period. In some cases, data transmitting requires the use of the entire bandwidth intensively. Numerous spectrum sharing methods were developed earlier. Some of them are carrier sense-based similar to CSMA/CD and CSMA/CA [1-4]. These methods 
are based on an assumption of network access stochastic nature. Their main drawback is a lack of performance while data rate increases. Medium availability decreases with the increasing of parallel sessions number. On the other hand, there is well-known group of dynamic deterministic medium sharing methods which use a software control for the purpose of spectrum allocation. Unlike the previously mentioned stochastic methods, the problem with deterministic methods is with the effectiveness of spectrum allocation under peak load. In the case of IoT devices, centralized spectrum allocation solution might be ineffective, and for such cases there is a need for a distributed approach to solving allocation problem.

Based on the research made in $[5,11]$ we achieved the increasing in energy effectiveness of IoT system based on routing cloudification approach. However, we did not consider the problem of the efficient medium access control in these papers. There are some research papers in the area of embedded system energy efficiency optimization for IoT devices by automatic switching to energy saving mode [6]. Moreover, we consider duration of medium access as vital for IoT device activity and, thus, for energy consumption. The problems of dynamic spectrum sharing, QoS-based spectrum channel allocation, spectrum sensing with distributed coordination are widely discussed in the papers authored by Z. Chkirbene I. F. Akyildiz et al. [7, 9, 12-14]. Y. Liu, K.-F. Tong, X. Qiu, Y. Liu Ta X. Ding [10] proposed the model of dynamic capacity allocation based on 802.11 DCF. This model estimates the number of user equipment allowed for a given spectrum range. However, in our opinion, this model could be considered as too idealistic.

Considering the state of the art review, we proposed our method of time distributed media access control for IoT devices.

\section{FUNDAMENTALS OF THE METHOD OF TIME DISTRIBUTED MEDIA ACCESS CONTROL FOR IOT DEVICES}

\section{A. THEORETICAL ASPECT FOR SUB-CARRIER SELECTION BY TWO IOT NODES}

For convenience, we treat bandwidth as the group of subcarries, called tunnels. A node is a computing unit along with an attached transceiver that can asynchronously change tunnels and modes (to transmit or to poll a chosen tunnel). Each node that participates in the link creation, sequentially stuff, and polls each sub-carrier one by one [15-17]. The procedure completes as a node 1 finds an available subcarrier and establishes connection with the node 2 , which responds to a connection request.

The main idea is that both node 1 and node 2 transmit and receive information sequentially through available tunnels. Hence, each node should transmit into all tunnels and try to receive from all tunnels one by one. According to this supposition on the side of a separate node, the process of picking tunnel is divided into time slots (TS), as shown in Fig. 1. During one TS, the node has two periods: integral transmission, i.e., the period of broadcasting into a tunnel; and polling, i.e., period, which is divided into several separate smaller receiving periods. The polling period is divided into several receives to create an artificial difference between phase velocities of processing transmits and receives. The phase velocity in this context means the tunnels iteration span or, in other words, the number of processed tunnels in constant time. Thus, if we got a set of available tunnels, e.g., 125, and distribute them equally along the cycle, imagine two arrows: one for the current transmit tunnel (TTA), and one for the current receive tunnel (RTA). When we have a difference in the phase velocity, the TTA will rotate a bit slower than RTA. For example, if the ratio is 2, one TX cycle takes the same time as two RX cycles.

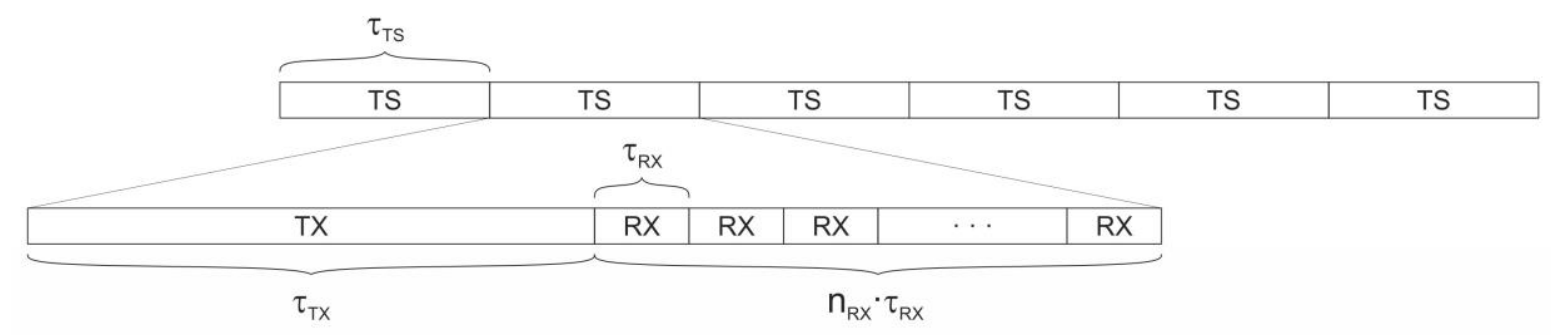

Figure 1. Structure of the time slot

And finally, let us clarify what we mean by transmit and receive periods. Transmit period (TX) is the period, during which node multiple times transmits a special sequence and waits for an acknowledgement. Receive period (RX) is the period, during which node polls the tunnel trying to catch the special sequence and to send an acknowledgement in case of success. Transmits and receives are performed according to ARQ SAW transmission mode [4].

Let us underline that the effective handshake duration for the ARQ SAW mode $\tau_{\text {Hands }}$ is directly depends on the bandwidth of sub-carrier and node's transceiver; quantity of available tunnels $N$ depends on the total bandwidth, apportioned for the system. Transsiver produces a delay $\tau_{\text {ChMod }}$, whilst switching between TX and RX modes. Let us assume that the time to change the mode from TX to RX equals the time to change the mode backwards.

Another benefit of the proposed method is an ability to control the interaction of IoT nodes and perform their clustering based on special identifiers. as shown in Fig. 2. Thus, every message sent by IoT node contains the cluster 
identifier field (ID), which depicts the belonging to a particular cluster. This information allows nodes to make local decisions regarding the joining in the cluster or setting up a connection with other node within this cluster. This approach simplifies IoT nodes group allocation to the cluster and configuration of their interaction rule set by reducing the chaotic nature of IoT communication. For example, nodes with a lack of resources could be set to respond to messages originated by another nodes within the same cluster only (same cluster ID) which leads to intra-cluster communication only. At the same time, more capable nodes will be able to fulfil the inter-cluster communication playing the role of so called superpeers.

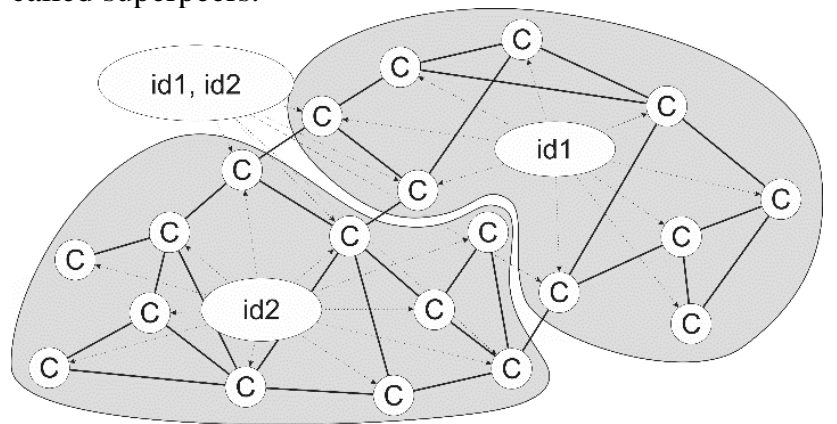

Figure 2. Two adjacent clusters: $\mathrm{msg} 1$ and $\mathrm{msg} 2$ are the special sequences for the first and second cluster, msg3 is a sequence for cluster-edge connections

Based on the mentioned assumptions, duration of an RX sub-slot $\tau_{R X}$ consists of two periods:

$$
\tau_{R X}=\tau_{\text {ChMod }}+\tau_{\text {Hands }},
$$

where $\tau_{\text {ChMod }}$ is the time that transceiver needs to switch the transmission mode (TX/RX) and sub-carrier, $\tau_{\text {Hands }}$ is the time that is needed to establish potentially successful message transmission between two nodes

Duration of the TX sub-slot $\tau_{T X}$ also consists of two periods:

$$
\begin{aligned}
& \tau_{T X}=\tau_{\text {ChMod }}+n_{R X} \cdot\left(\tau_{\text {ChMod }}+\tau_{\text {Hands }}\right)= \\
& =\tau_{\text {ChMod }}+n_{R X} \tau_{R X}, n_{R X} \in(0 \ldots N)
\end{aligned},
$$

where $n_{R X}$ is the quantity of RX periods inside one TS (Fig. 2), $N$ is the total number of sub-carriers in the dedicated spectrum

Hence, the duration of the TS $\tau_{T S}$ is:

$$
\tau_{T S}=\tau_{T X}+n_{R X} \tau_{R X}
$$

As we can observe in Fig. 3 maximal TS coverage can be reached when the duration of TX equals to the sum for $\mathrm{N}$ durations of RX:

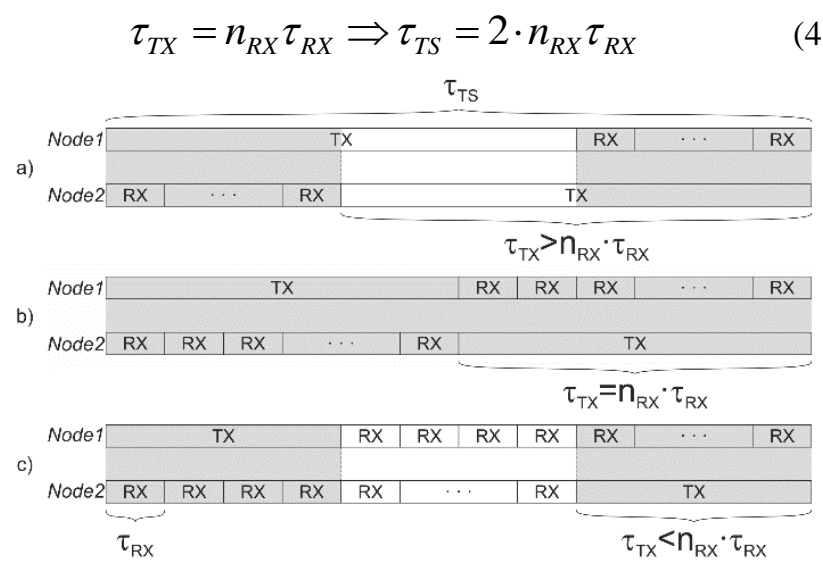

Figure 3. The influence of the ratio between the broadcasting and polling period durations. If the durations are different, "blind zones" occur $(\mathrm{a}, \mathrm{c})$ and some part of TS is not able to commit a transaction. In contrast, if they are equal the TS is used entirely (b)

Now given the bounding between $\tau_{T X}$ and $\tau_{R X}$, their durations can be solved relative to the $\tau_{T S}$, e.g. taking that $\tau_{T S}=1$ :

$$
\begin{gathered}
\tau_{R X}=\frac{\tau_{T S}}{2 n_{R X}}=0.5 \cdot n_{R X} . \\
\tau_{T X}=\frac{\tau_{T S}}{2}=0.5 .
\end{gathered}
$$

\section{B. SHIFT-LESS OPERATION}

For the case considered above, two operation modes exist. The first mode assumes the external synchronization of the nodes, so called mode with coordinated TS's. In this case, when the first node is starting its TX period, the other one immediately starts the RX period, as shown in Fig. 3 (b). Theoretically, this is the perfect case, but it is possible only when nodes are synchronized by some intermediator. So, if the TS of node 1 is synchronized with the one of a node 2 , the formula for the average TS number $n_{\text {TSexec }}$ that is needed to successfully find the appropriate tunnel and accomplish handshake between nodes is:

$$
n_{\text {TSexec }}\left(n_{R X}\right)=1+\frac{N-n_{R X}}{n_{R X}-1}-\frac{1}{\sqrt{2}} \cdot \frac{N-n_{R X}}{n_{R X}-1}
$$

Given the chosen duration of one TS $\tau_{T S}$, the average time needed to accomplish handshake $\tau_{\text {exec }}$ in case of 
fixed $N$ is:

$$
\begin{aligned}
& \tau_{\text {exec }}\left(n_{R X}\right)=\tau_{T S} n_{T S e x e c}\left(n_{R X}\right)= \\
& =\frac{\tau_{T S}}{2} \cdot\left(\frac{N(2-\sqrt{2})-2+\sqrt{2} \cdot n_{R X}}{n_{R X}-1}\right)
\end{aligned}
$$

Let us consider the following simplifications: spectrum is free along with the dedicated bandwidth (100/100 tunnels are free and ready to be occupied), the SNR is infinite (100/100 bits are transmitted correctly) [19].

Fig. 4 depicts the relation between the TS number mean value just before the connection was established, and $n_{R X}$ for the system containing 2 processes and the total number of the tunnels $N=200$ which they both have for their disposal.

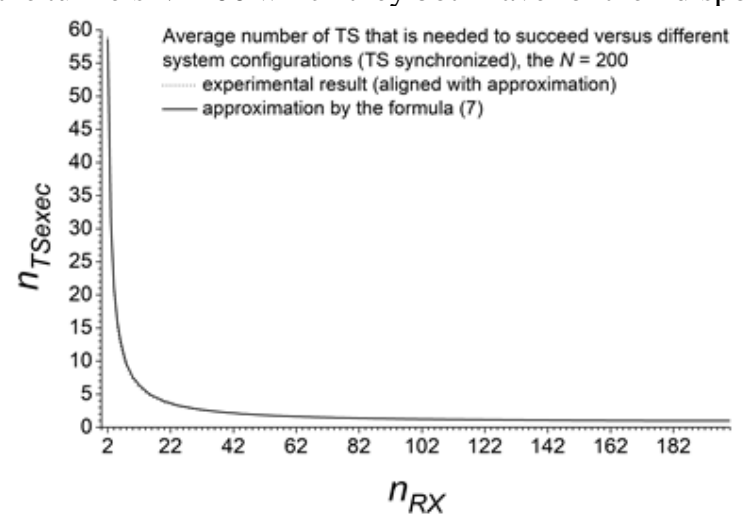

Figure 4. The dependency of an average number of TS before connection happens versus the number of RX sub-slots for 1 TX slot (total number of given tunnels is 200)

Let us highlight that $n_{R X}$ increasing causes the $\tau_{T S}$ growth, and based on that we could formulate the relation between a mean connection delay timeout $\tau_{\text {exec }}\left(n_{T S}\right)$ and $n_{R X}$. This relation allows us to determine the optimal number of $n_{R X}$, which corresponds to the minimal $\tau_{\text {exec }}\left(n_{T S}\right)$ (Fig. 5, $n_{R X}=9$ corresponds to the minimal $\left.\tau_{\text {exec }}\left(n_{T S}\right)\right)$.

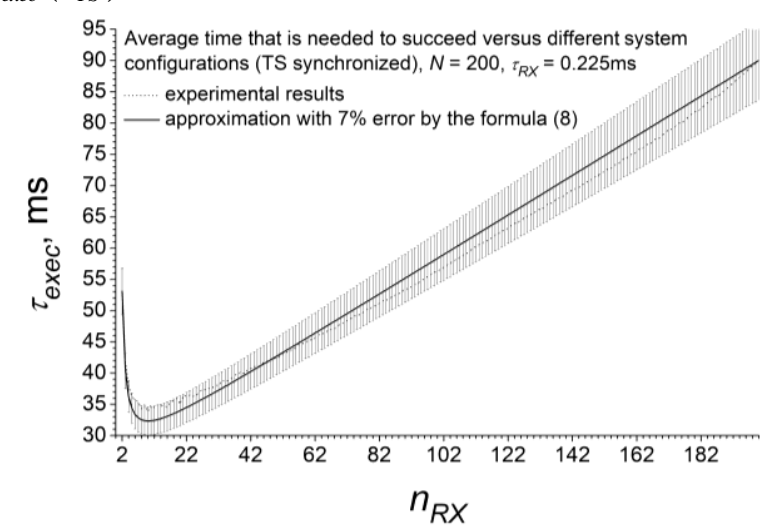

Figure 5. Mathematical approximation, formula (8), and experimental modelling results for the system with $0.225 \mathrm{~ms}$ RX sub-slot (total number of given tunnels is 200)

\section{SHIFT-FULL OPERATION}

Let us consider a second mode which is a bit more precise and describes the real systems behavior. Here the TS's are not synchronized, i.e., can be shifted in time $\tau_{\text {Shift }}$ by several RX sub-slots plus a certain amount of indivisible time, which is less than a duration of the RX sub-slot. Unfortunately, this shift between TS's harm the average time needed to choose the tunnel and perform a handshake. This is related with a limited capacity of the tunnel (Fig. 6). This mode is especially actual for the inter-cluster interaction, for which limited tunnel capacity causes so called blinded zones between the pair of the IoT nodes. In such zones both nodes simultaneously have either TX sub-slot or RX sub-slot. The resulting shift $n_{\text {Shift }}$, expressed in the number of RX subslots is:

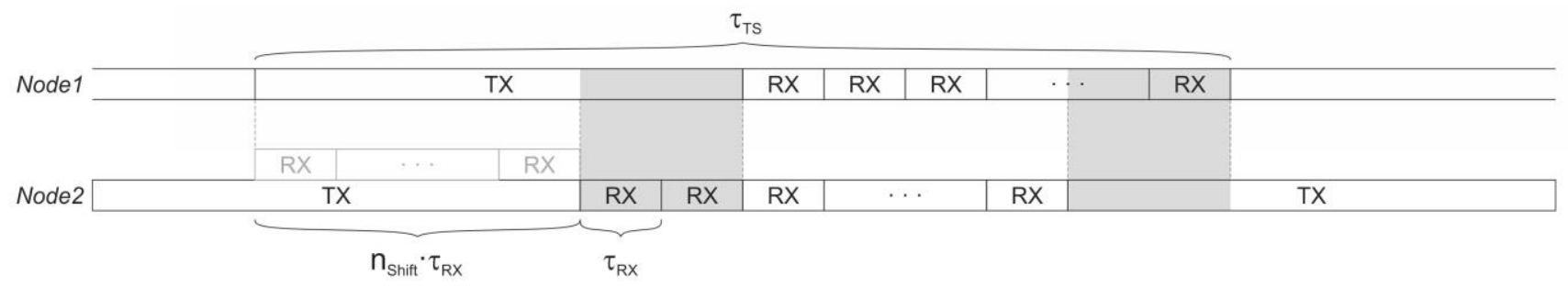

Figure 6. TS of a node 1 is shifted in time relatively to the TS of a node 2, thus, decreasing the region, where handshake can happen

$$
n_{\text {Shift }}=\left\lceil\frac{\tau_{\text {Shift }}}{\tau_{R X}}\right\rceil
$$

Assume that the absolute shift value can be any value between $\mathbf{O}$ and $\tau_{T S}$. Thus the average number of an active
RX sub-slots $n_{R X}^{\prime}$ equals the number of RX sub-slots in TS minus the average number of RX sub-slots $n_{\text {Shift }}^{\prime}$ that are lost due to the shift:

$$
n_{R X}^{\prime}=n_{R X}-n_{\text {Shift }}^{\prime}=n_{R X}-\sum_{n_{\text {Shift }}=1}^{n_{R X}} \frac{n_{\text {Shift }}}{n_{R X}}
$$


Hence, the formula for an average number of TS $n_{\text {TSexec }}^{\prime}$ before node successfully chooses tunnel and performs handshake is:

$$
n_{\text {TSexec }}^{\prime}\left(n_{R X}\right)=\frac{N \cdot\left(1-\frac{1}{\sqrt{2}}\right)+\frac{n_{R X}}{\sqrt{2}}-\frac{1}{\sqrt{2}} \sum_{n_{\text {Shift }}=1}^{n_{R X}-3} \frac{n_{\text {Shift }}}{n_{R X}}}{n_{R X}-\sum_{n_{\text {Shift }}=1}^{n_{R X}-3} \frac{n_{\text {Shift }}}{n_{R X}}-1}
$$

Also, we should consider one important case: what if there is such a shift, that the whole TS is under a blind zone, and it's impossible to perform the handshake. This means that there is a small probability $p_{\text {Inf }}$ of not ever establishing a connection:

$$
p_{\text {Inf }}\left(n_{R X}\right)=\frac{1}{n_{R X}+1}
$$

However, such cases can be mitigated by introducing

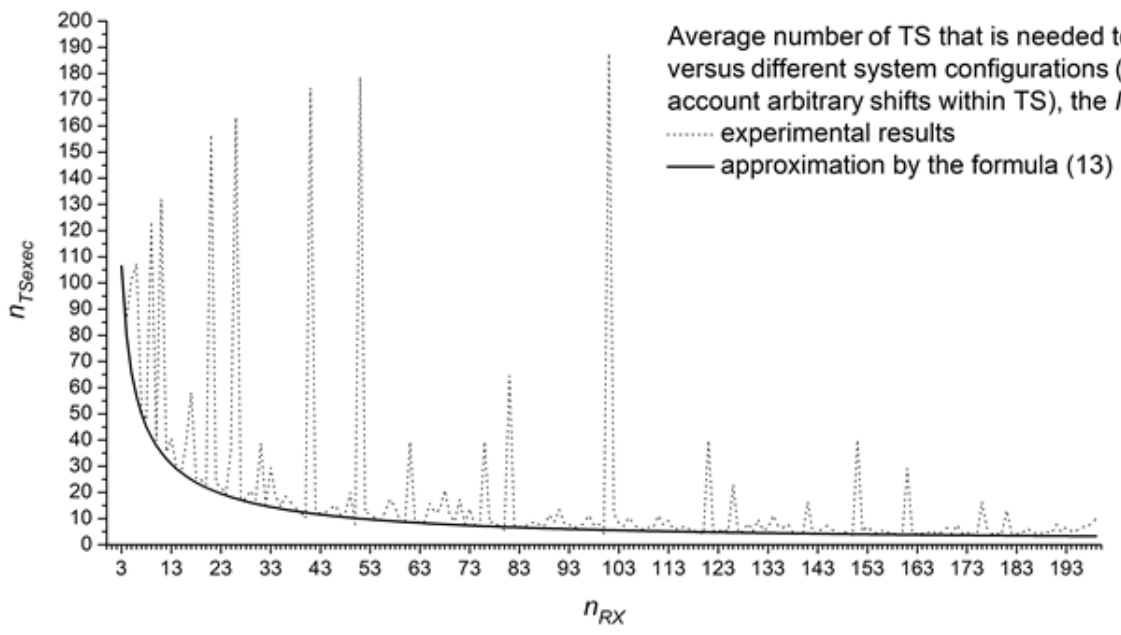

Figure 7. Mathematical approximation, formula (13), and experimental modelling results for the system with arbitrary shifts within TS (total number of given tunnels is 200)

As can be noted from the plot, the formula (13) approximates most of the experimental curve, though huge surges are present. These surges reflect cases, where the total number of tunnels in the system is directly divisible by the number of RX sub-slots per $1 \mathrm{TX}$ sub-slot. Also, smaller surges show cases, where a certain value exists so that the total number of tunnels in the system, as well as the number of RX sub-slots per one TX sub-slot, are directly divisible by it. As a result, depending on these specific parameters, the system can perform better or worse. Let us consider the following restrictions on choosing the parameters for each specific system: periodic random impediments. The duration of the impediment must be chosen between $\mathbf{O}$ and the $\frac{1}{2} \tau_{T S}$. Using this technique, in the worst-case nodes, should decrease the blind zone as soon as they separately perform these random impediments. Finally, the resulting formula for an average number of TS $n_{T S e x e c}^{\prime \prime}$ needed to successfully choose the appropriate tunnel and perform handshake is:

$$
\begin{aligned}
& n_{\text {TSexec }}^{\prime \prime}\left(n_{R X}\right)=\frac{N \cdot\left(1-\frac{1}{\sqrt{2}}\right)+\frac{n_{R X}}{\sqrt{2}}-\frac{1}{\sqrt{2}} \sum_{n_{\text {Shift }}=1}^{n_{R X}-3} \frac{n_{\text {Shift }}}{n_{R X}}}{n_{R X}-\sum_{n_{\text {Shift }}=1}^{n_{R X}-3} \frac{n_{\text {Shift }}}{n_{R X}}-1} * \\
& *\left(1+\sum_{i=1}^{\infty} p_{\text {Inf }}^{i}\left(n_{R X}\right)\right)+\left(N+\frac{1}{4}\right) \cdot \sum_{i=1}^{\infty} p_{\text {Inf }}^{i}\left(n_{R X}\right)
\end{aligned}
$$

This formula approximates the process of establishing a connection and considers everything that we have mentioned (Fig. 7).

\section{TS that is needed to succeed} versus different system configurations (taking into count arbitrary shifts within TS), the $N=200$ ..... experimental results

approximation by the formula (13)

$$
\left\{\begin{array}{c}
\frac{[N-\xi \cdots N+\xi]}{n_{R X}} \notin \mathrm{N} \\
{\left[\left(\frac{[N-\xi \cdots N+\xi]}{i} \notin \mathrm{N}\right) \cap\left(\frac{n_{R X}}{i} \notin \mathrm{N}\right)\right], i \in\left(0 \ldots n_{R X}\right)}
\end{array}\right.
$$

where $i$ is a natural value, it determines how much the results can be approximated by the formula (13), i.e., the areas without local peaks.

These peaks nature could be explained based on Fig. 8 . As we can see, because of a shift, the certain group of tunnels will stay in the blind zone forever, such as tunnels $3,4,9,10$, 15,16 , and that is why the total quantity of available tunnels in the system decreases and its characteristics change dramatically. Moreover, shifts introduce asymmetry, which makes it a bit complex task to analyze such systems. 


\begin{tabular}{|c|c|c|c|c|c|c|c|c|c|c|}
\hline & & & & & & & & & & \\
\hline & 13 & 14 & 15 & 16 & 17 & 18 & \\
\hline & & & & 7 & 8 & 9 & 10 & 11 & 12 & \\
\hline Node1 & $\mathrm{TX}$ & & & 1 & 2 & 3 & 4 & 5 & 6 & \\
\hline \multirow[t]{3}{*}{ Node2 } & & 13 & 14 & 15 & 16 & 17 & 18 & & & \multirow[t]{3}{*}{ TX } \\
\hline & & 7 & 8 & 9 & 10 & 11 & 12 & & & \\
\hline & & 1 & 2 & 3 & 4 & 5 & 6 & & & \\
\hline
\end{tabular}

Figure 8. A system with the 18 tunnels in total and $6 \mathrm{RX}$ sub-slots for $1 \mathrm{TX}$ slot, numbers inside sub-slots indicate corresponding tunnels, which are served by that sub-slots in different cycles. As 18 is directly divisible by 6 , and the result of such division is 3, each third TS will contain the same tunnels on the same positions. After introducing the shift, the situation occurs, where certain tunnels will stay in the blind zone forever.

Finally, given that the system was designed using the restrictions (14), the average time for connection establishment $\tau_{\text {exec }}^{\prime \prime}$ can be calculated as:

$$
\tau_{\text {exec }}^{\prime \prime}\left(n_{R X}\right)=\tau_{T S} n_{T S e x e c}^{\prime \prime}\left(n_{R X}\right)
$$

The resulting plot (Fig. 9), in contrast to the previous plots, was presented for the system with the total number of tunnels 199, which is a prime number. The prime number was chosen because it is divisible only by 1 and itself. The surges on the curve are explained by the fact, that values around 199 exist and are divisible by the number of RX subslots per 1 TX sub-slot. Examples are 196, 197, 198 on the left-hand side and 200, 201, 202 on the right-hand side. Nonlinear boundaries (very small and large number of RX sub-slots per $1 \mathrm{TX}$ sub-slot) on the plot are vagueness regions, a.k.a. boundary conditions, and are out of this paper focus.

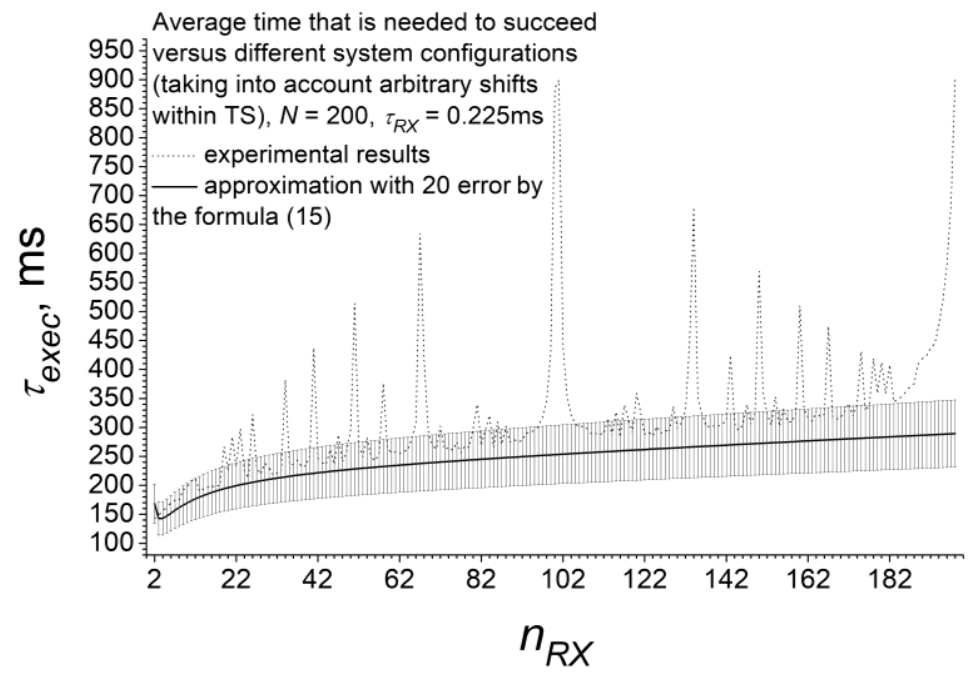

Figure 9. Mathematical approximation, formula (15), and experimental modelling results for the system with arbitrary shifts within TS and $0.225 \mathrm{~ms}$ RX sub-slot (total number of given tunnels is 199)

\section{CONCLUSIONS}

Today's techniques, such as CSMA or NB-IoT, use either sequential intense access to the spectrum or an arbiter approach. In contrast, the technique, which is described in this work, proposes another method for deploying mesh networks without an explicit radio resource controlling system that is applicable for typical IoT applications. The dedicated spectrum is divided into a set of tunnels that can be used as P2P links. In the paper, we presented a newly developed mechanism for choosing an appropriate tunnel for a P2P link. Devices can use it to choose free tunnels and establish links between each other. Hence, the spectrum access is automatically controlled by IoT units themselves.
We have studied different configurations for the presented method and built mathematical approximations to simulate the behaviour of the corresponding system. This allows an external researcher to build a similar system and choose corresponding parameters for better performance. If the system is perfect, and TS of separate devices are perfectly aligned, the analysis results showed that around $9 \mathrm{RX}$ subslots within TS work better for the system with 200 subcarriers in total. Also, results showed that approximation, in this case, is accurate, i.e., we got maximum error of $7 \%$. However, when considering that TS can start in different moments for different nodes, the behaviour of the system is more complex. Though in this scenario system is more stable and does not depend on an external synchronizer. The results 
showed that extremely inefficient configurations are possible (surges on the plot, Fig. 6). To avoid such configurations while developing a similar CR system, one can use the block of conditionals (14). Also, the majority of the experimental results fits into a $20 \%$ error bar which states that our approximation, formula (15), can be applied to predict the average time of establishing a connection between nodes and, as a result, to build appropriate system designs. For a system with 200 sub-carriers available, the best configuration is $3 \mathrm{RX}$ sub-slots, and the average time for linking does not differ much from configuration to configuration, given that block of conditionals (14) is satisfied.

In further research, we plan to prove the energy efficiency of the proposed method of access to spectrum resources and its practical implementation on the example of $5 \mathrm{G}$ networks.

\section{References}

[1] W. Xiaofan, P. H. J. Chong, and L. W. Yie, "Performance comparison of CSMA/CD, CSMA/CA, CSMA/RI, CSMA/PRI and CSMA/PR with BEB," Proceedings of the 2010 5th IEEE Conference on Industrial Electronics and Applications, ICIEA 2010, Taichung, 2010, pp. 1843-1848, https://doi.org/10.1109/ICIEA.2010.5515401.

[2] A. Nasipuri, J. Zhuang, and S. R. Das, "A multichannel CSMA MAC protocol for multihop wireless networks," Proceedings of the IEEE Wireless Communications and Networking Conference, WCNC'1999, New Orleans, LA, USA, 1999, vol. 3, pp. 1402-1406. https://doi.org/10.1109/WCNC.1999.796968.

[3] D. Miorandi, S. Sicari, F. De Pellegrini, and I. Chlamtac, "Internet of things: Vision, applications and research challenges," Ad Hoc Networks. vol. 10, issue 7, pp. 1497-1516, 2012. https://doi.org/10.1016/j.adhoc.2012.02.016.

[4] I. Demirkol, C. Ersoy, and F. Alagöz, "MAC protocols for wireless sensor networks: A survey," IEEE Commun. Mag., vol. 44, no. 4, pp. 115-121, 2006, https://doi.org/10.1109/MCOM.2006.1632658.

[5] V. Faychuk, O. Lavriv, B. Strykhalyuk, O. Shpur, I. Demydov, and R. Bak, "Performance of routing algorithm remote operation in cloud environment for IoT devices," Int. J. Electron. Telecommun., vol. 65 , no. 3, pp. 367-373, 2019. https://doi.org/10.24425/ijet.2019.129787.

[6] G. Anastasi, M. Conti, M. Di Francesco, and A. Passarella, "Energy conservation in wireless sensor networks: A survey," Ad Hoc Networks, vol. 7, issue 3, pp. 537-568, 2009. https://doi.org/10.1016/j.adhoc.2008.06.003.

[7] Z. Chkirbene and N. Hamdi, "A survey on spectrum management in cognitive radio networks," Int. J. Wirel. Mob. Comput., vol. 8, no. 2, 153-165, 2015, https://doi.org/10.1504/IJWMC.2015.068618.

[8] G. Bianchi, "Performance analysis of the IEEE 802.11 distributed coordination function," IEEE J. Sel. Areas Commun., vol. 18, no. 3, pp. 535-547, 2000. https://doi.org/10.1109/49.840210.

[9] I. F. Akyildiz, W. Y. Lee, and K. R. Chowdhury, "CRAHNs: Cognitive radio ad hoc networks," Ad Hoc Networks, vol. 7, no. 5, pp. 810-836, 2009, https://doi.org/10.1016/j.adhoc.2009.01.001.

[10] Y. Liu, K.-F. Tong, X. Qiu, Y. Liu, and X. Ding, "Wireless mesh networks in IoT networks," Proceedings of the 2017 International Workshop on Electromagnetics: Applications and Student Innovation Competition, 2017, pp. 183-185. https://doi.org/10.1109/iWEM.2017.7968828.

[11] V. Faychuk, O. Lavriv, M. Klymash, V. Zhebka, and O. Shpur, "Investigation of the Bellman-Ford algorithm enhanced for remote execution," Proceedings of the 2019 3rd International Conference on Advanced Information and Communications Technologies, AICT'2019, Lviv, Ukraine, 2019, pp. 204-208. https://doi.org/10.1109/AIACT.2019.8847727.

[12] M. Shirkhani, Z. Tirkan, and A. Taherpour, "Performance analysis and optimization of two-way cooperative communications in intervehicular networks," Proceedings of the 2012 International
Conference on Wireless Communications and Signal Processing, WCSP'2012, 2012, 2 pp. https://doi.org/10.1109/WCSP.2012.6542974.

[13] O. B. Akan, O. B. Karli, and O. Ergul, "Cognitive radio sensor networks," IEEE Netw., vol. 23, no. 4, pp. 34-40, 2009. https://doi.org/10.1109/MNET.2009.5191144.

[14] G. P. Joshi, S. Y. Nam, and S. W. Kim, "Cognitive radio wireless sensor networks: Applications, challenges and research trends," Sensors (Switzerland), vol. 13, issue 9, pp. 11196-11228, 2013. https://doi.org/10.3390/s130911196.

[15] Y. Zeng and Y. C. Liang, "Eigenvalue-based spectrum sensing algorithms for cognitive radio," IEEE Trans. Commun., vol. 57, no. 6, pp. 1784-1793, 2009. https://doi.org/10.1109/TCOMM.2009.06.070402.

[16] K. Kredo and P. Mohapatra, "Medium access control in wireless sensor networks," Comput. Networks, vol. 51, issue 4, pp, 961-994, 2007. https://doi.org/10.1016/j.comnet.2006.06.012.

[17] I. F. Akyildiz, W. Y. Lee, M. C. Vuran, and S. Mohanty, "NeXt generation/dynamic spectrum access/cognitive radio wireless networks: A survey," Comput. Networks, vol. 50, issue 13, pp. 2127 2159, 2006. https://doi.org/10.1016/j.comnet.2006.05.001.

[18] V. M. Patil and S. R. Patil, "A survey on spectrum sensing algorithms for cognitive radio," Proceedings of the 2016 International Conference on Advances in Human Machine Interaction, HMI'2016, 2016, pp. 1-5. https://doi.org/10.1109/HMI.2016.7449196.

[20] T. Sowlati and D. M. W. Leenaerts, "A 2.4-GHz 0.18- $\mu \mathrm{m}$ CMOS selfbiased cascode power amplifier," IEEE J. Solid-State Circuits, vol. 38 no. $8, \quad$ pp. 2003. https://doi.org/10.1109/JSSC.2003.814417.

[21] Nordic, "nRF24L01+ single chip $2.4 \mathrm{GHz}$ transceiver product specification v1.0," Building Research and Information, vol. 21, issue $\begin{array}{llll}1, & \text { pp. } & 21-22, & 1993 .\end{array}$ https://doi.org/10.1080/09613219308727250.

[22] J. Mitola and G. Q. Maguire, "Cognitive radio: making software radios more personal," IEEE Pers. Commun., vol. 6, no. 4, pp. 13-18, 1999. https://doi.org/10.1109/98.788210.

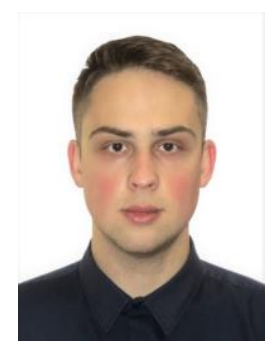

VALENTYN FAYCHUK, Student of the Department of Telecommunications at Lviv Polytechnic National University. His research interests: loT, resources allocation and access, control systems, distributed services.

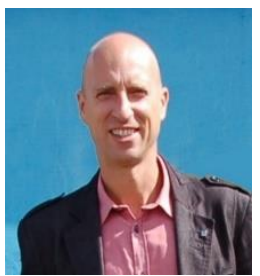

DARIUSZ MROZEK, currently an Associate Professor and Head of the Department of Applied Informatics at the Silesian University of Technology (SUT) in Gliwice, Poland. Research interests: cover Internet of Things, bioinformatics, information systems, parallel and Cloud computing and big data.

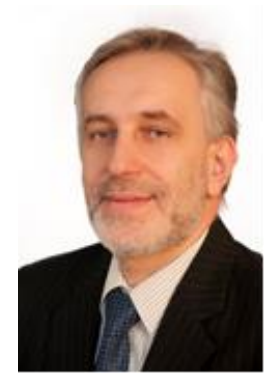

BOHDAN STRYKHALIUK, Doctor of Science, Professor of the Department of Radio-Electronic Devices and Systems at Lviv Polytechnic National University. His research interests: include theoretical foundations of the construction and operation of next generation networks and cloud, IOT technology 


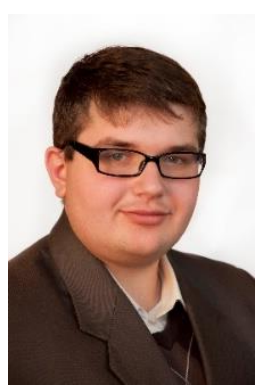

OREST LAVRIV, Doctor of science, Lecturer of the Department of ELECTRONICS and Information Technology at Lviv Polytechnic National University. His research interests: service engineering, 5G, SDN, IOT Routing Cloudification, Cloud

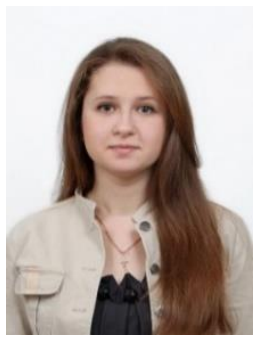

Olga Shpur, PhD, Associate Professor of the Department of Telecommunications at Lviv Polytechnic National University. Her research interests include design features and operation of networks based on service-oriented architectture, cloudtechnology, SDN. 